\title{
Highlights from the American society of hematology conference 2020
}

\author{
Punita Grover ${ }^{1} \cdot$ Shuchi Gulati ${ }^{1}[$ \\ Accepted: 13 April 2021 / Published online: 26 April 2021 \\ (c) The Author(s), under exclusive licence to Springer Science+Business Media, LLC, part of Springer Nature 2021
}

\begin{abstract}
American Society of Hematology conducts an annual meeting, where investigators from around the globe presented groundbreaking research in the fields of malignant and non-malignant hematology. We provide a summary of non-malignant hematology abstracts from the 2020 meeting. Topics included range from those related to thrombosis, including thrombotic complications of COVID-19, bleeding and novel therapies such as gene therapies. Readers are encouraged to access meeting materials for a more detailed coverage of the event.
\end{abstract}

Keywords ASH2020 · Thrombosis · COVID-19 · Bleeding

\section{Introduction}

The American Society of Hematology (ASH) Annual Meeting 2020 had several exciting presentations in non-malignant hematology. Here we focus on a few high impact and practice-changing presentations. The review will be divided into three sections-thrombosis (including COVID-19 related thrombosis); bleeding; gene therapy in non-malignant hematology.

\section{Thrombosis}

\section{Safety and efficacy of the contact activation inhibitor AB023 in patients with end-stage renal disease (ESRD) on chronic hemodialysis (HD): a phase 2, double-blind, randomized, placebo-controlled trial}

Clot formation in HD circuits can result in complications such as blood loss and decreased efficiency of the dialysis units or failure. [1] Exposure of blood to artificial surfaces within the HD circuit or other life preserving devices activates factor XII, which initiates the contact-activation coagulation pathway. $\mathrm{AB} 023$, a humanized $\mathrm{IgG} 4$ antibody, binds to the A2 domain of Factor XI (FXI), thereby inhibiting the activation of FXI by FXII and hence the contact-activation pathway but does not interfere with thrombin-mediated FXI activation. A randomized phase- 2 study enrolled 24 patients with ESRD on chronic HD to receive a single dose of AB023 ( 0.25 or $0.5 \mathrm{mg} / \mathrm{kg})$ vs. placebo into the HD circuit at the start of heparin free HD. [2] The frequency of occlusive events requiring circuit exchange decreased by $68 \%$ in patients given $0.25 \mathrm{mg} / \mathrm{kg}$ and by $50 \%$ with $0.5 \mathrm{mg} /$ $\mathrm{kg} \mathrm{AB023,} \mathrm{while} \mathrm{it} \mathrm{remained} \mathrm{unchanged} \mathrm{in} \mathrm{the} \mathrm{placebo} \mathrm{arm.}$ There was a significant decrease in the number of saline flushes required to maintain circuit patency ( $44 \%$ and $85 \%$ with 0.25 and $0.5 \mathrm{mg} / \mathrm{kg}$ respectively). The incidence of high-grade clotting in the hemodialyzer assessed by visual scoring also decreased in a dose-dependent manner. AB023 reduced thrombin generation as detected by thrombin antithrombin complexes measured four hours after dosing. No drug-related adverse events (AEs) were noted, including clinically relevant bleeding. The time to hemostasis at the HD access site did not change after AB023 administration.

The results of this trial are encouraging because the efficacy of selective FXI antagonism and inhibition of contact system activation could prevent device-associated thrombosis.

Shuchi Gulati

gulatisi@ucmail.uc.edu

1 Division of Hematology and Oncology, University of Cincinnati, Cincinnati, OH 45267, USA 


\section{Prior history of venous thromboembolism is a significant risk factor for recurrence of thrombosis after cancer diagnosis}

Patients with cancer have an increased risk of thrombosis resulting in treatment delays, increased morbidity and mortality. The Khorana risk score is a validated venous thromboembolism (VTE) prediction tool, which guides the use of prophylactic anticoagulation in ambulatory patients with cancer. [3] Prior history of VTE is a known risk factor for recurrent events in the general population [4] but this variable is not included in the Khorana risk score.

A retrospective cohort study using the IBM Watson Explorys electronic health record database was conducted to analyze the effect of prior VTE on the incidence of cancer-associated VTE. [5] A total of 4,159,400 patients with cancer were identified, of whom $3.3 \%(n=138,820)$ had a history of VTE $>1$ year prior to being diagnosed with cancer. There was a tenfold higher incidence of cancer associated VTE at 6 months in those with a prior history of VTE compared to those without [36.9\% vs. $3.66 \%$; OR 15.4 (95\% CI 15.22-15.6), $\mathrm{p}<0.0001]$. This increased risk was independent of established risk factors, cancer type or Khorana risk score. Most of the VTE events occurred within the first month of cancer diagnosis. Patients with a cancer associated VTE who had a prior VTE were more likely to be obese, receiving antineoplastic treatment and have Factor V Leiden mutation or activated protein $\mathrm{C}$ resistance.

This is the first large study to quantify the risk of cancer associated VTE in patients with prior VTE. Being a retrospective study, it had several limitations-specifically information about cancer stage, surgery, treatment intent, provoked/unprovoked nature of prior VTEs and anticoagulation was not available. Large prospective observational studies would be needed to validate these findings.

\section{Direct oral anticoagulants (doacs): dosing, complications and reversal}

\section{Australian and New Zealand registry of anticoagulation in the obese: prescribing patterns, drug levels and patient outcomes}

Obesity is rapidly increasing worldwide and is a known risk factor for VTE. [6] Obesity can affect drug pharmacokinetics and there is limited data guiding the dosing of available anticoagulants in obese patients. Key randomized control trials comparing DOACs with LMWH/warfarin did not include patients at extremes of weight.
The study presented at ASH, 2020 was a multicenter, prospective trial which enrolled 155 patients with BMI $>35 \mathrm{~kg} / \mathrm{m}^{2}$ or weight $>120 \mathrm{~kg}$ who were diagnosed with VTE and treated with various anticoagulants. [7] Median age was 53 years (range: $22-85$ ), $49 \%$ were male, $77(50 \%)$ had pulmonary embolism (PE) and $62(40 \%)$ had deep vein thrombosis (DVT) without PE. 37 (24\%) received apixaban, 57 (37\%) received rivaroxaban, 29 (19\%) received LMWH and $28(18 \%)$ received warfarin (Table 1).

The mean weight and BMI of patients treated with a DOAC was significantly lower than those treated with LMWH or warfarin (127 vs. $152 \mathrm{~kg}$, BMI: 44.6 vs. $50 \mathrm{~kg} /$ $\mathrm{m}^{2}$ ). All peak apixaban levels $(\mathrm{n}=15)$ and $68 \%(25$ of 37$)$ of peak rivaroxaban levels were within therapeutic range. The assessment of trough levels could not be performed due to the limit of detection at different laboratories. 22 of 28 patients on LMWH had peak anti-Xa within the target range and the median enoxaparin dose to achieve this was $0.9 \mathrm{mg} / \mathrm{kg}$ (range $0.52-1.3$ ). Two patients on DOACs had recurrent $\mathrm{PE}$, both in the setting on non-compliance while one patient on LMWH had an equivocal recurrence despite the presence of a supra-therapeutic anti Xa.

Even though this study is limited by small numbers and lack of standardized follow-up, it adds to a growing body of literature demonstrating the safety and efficacy of DOACs in obese patients. Monitoring DOAC drug levels is recommended for obese patients but is challenging due to lack of widespread availability of the assay and limited data on dose modifications based on drug levels. The results of this study suggest that such monitoring may not be required, but the results will need to be confirmed in larger studies.

Table 1 Baseline characteristics of patients included in the Australian and New Zealand registry of anticoagulation in the obese [7]

\begin{tabular}{ll}
\hline Number of patients & 155 \\
Age (median, range) & $53(22-85)$ \\
Male & $76(49 \%)$ \\
Indication & \\
PE & $77(50 \%)$ \\
DVT & $62(40 \%)$ \\
Initial anticoagulant & \\
Apixaban & $37(24 \%)$ \\
Dabigatran & $4(2.6 \%)$ \\
Rivaroxaban & $57(37 \%)$ \\
LMWH & $29(19 \%)$ \\
Warfarin & $28(18 \%)$ \\
Mean weight and BMI & \\
DOAC & $127 \mathrm{~kg}, 44.6 \mathrm{~kg} / \mathrm{m}^{2}$ \\
Warfarin or LMWH & $152 \mathrm{~kg}, 50.0 \mathrm{~kg} / \mathrm{m}^{2}$ \\
\hline
\end{tabular}




\section{Mortality rate and functional outcomes among patients presenting with anticoagulation-associated intracranial hemorrhage}

Intracranial hemorrhage is one of the most devastating complications in patients on anticoagulation and occurs at an annual rate of $0.6-0.7 \%$ in patients on prior warfarin and $0.1-0.2 \%$ in patients on DOACs. [8, 9] ICH is associated with high rates of mortality and disability but there is limited data on the clinical outcomes of patients with anticoagulant-associated ICH.

A retrospective study analyzing the in-hospital mortality rate and functional outcomes of patients with anticoagulant associated intracranial hemorrhage was presented at ASH, 2020. [10] A total of 1331 patients were admitted with ICH, 195 were on an oral anticoagulant (77 DOAC and 118 warfarin), 258 on antiplatelet monotherapy and 835 were not on anticoagulant/antiplatelet therapy preceding ICH. Patients on oral anticoagulant prior to $\mathrm{ICH}$ were older and the indication for anticoagulation was atrial fibrillation in majority of the patients $(81.8 \%$ in DOACs and $73.7 \%$ in warfarin). Patients who were not on prior anticoagulant/antiplatelet therapy had longer length of admission and stay in intensive care and were also more likely to undergo operative intervention (Table 2). Subdural hematomas were more common in patients on warfarin while intraparenchymal bleed was more frequent with DOACs. The average in-hospital mortality was higher with an oral anticoagulant-associated ICH $(37.7 \%$ with DOACs, $36.5 \%$ with warfarin) compared to $22.4 \%$ with antiplatelet monotherapy and $16.8 \%$ with no anticoagulant/ antiplatelet therapy. Among patients on DOACs, the inhospital mortality was $29 \%$ for those on apixaban $(n=31)$ and $46 \%$ for those on rivaroxaban $(n=39)$. The functional outcomes at discharge (measured by the modified Rankin Scale) showed moderate to severe disability in all groups.
The results of this study differ from other published studies which showed a lower rate of mortality with DOAC-associated ICH compared with warfarin-associated ICH. [11, 12] This study did not have details about the use of reversal agents or the outcomes of patients on both anticoagulant and antiplatelet therapy and prospective trials will need to be done to confirm these findings.

\section{Reversal of anticoagulation by ciraparantag: time to onset and duration of effect}

Ciraparantag is a unique anticoagulant reversal agent with broad activity. It is a small molecule that binds non-covalently to several anticoagulants including DOACs, LMWH, unfractionated heparin (UFH) without binding to endogenous coagulation factors or plasma proteins.

Two phase 2 dose-ranging studies were conducted in healthy volunteers $50-75$ years of age to assess the time to onset of anticoagulation reversal and duration of effect with ciraparantag. [13] 49 subjects who received apixaban and 64 who received rivaroxaban were randomized 3:1 to receive either a single dose of IV ciraparantag or placebo. Manual whole blood clotting time (WBCT) was performed at multiple time-points over $24 \mathrm{~h}$ and reversal was defined by decrease in WBCT to $\leq 10 \%$ above baseline. Traditional plasma-based assays (like aPTT, PT, anti-Xa) could not be used since ciraparantag binds to reagents (citrate and EDTA) in test-tubes rather than the anticoagulant in blood. Ciraparantag demonstrated a rapid and dose-dependent reversal of both apixaban and rivaroxaban. For apixaban, mean WBCT reversal to $\leq 10 \%$ above baseline, at 15 min postinfusion, occurred with 60 and $120 \mathrm{mg}$ ciraparntag while a higher dose of $180 \mathrm{mg}$ ciraparantag was needed for rivaroxaban. Approximately $80 \%$ of patients who had received apixaban achieved reversal at $15 \mathrm{~min}$ with $60 \mathrm{mg}$ ciraparantag, which increased to $100 \%$ at $60 \mathrm{~min}$. For subjects who had received rivaroxaban, $180 \mathrm{mg}$ ciraparantag lead to reversal
Table 2 Baseline characteristics and in-hospital morality among patients presenting with anticoagulation-associated intracranial hemorrhage [10]

\begin{tabular}{lllll}
\hline & $\begin{array}{l}\text { No anticoagulant } \\
\text { or antiplatelet }\end{array}$ & DOAC & Warfarin & $\begin{array}{l}\text { Antiplatelet } \\
\text { monother- } \\
\text { apy }\end{array}$ \\
\hline Number of patients & 835 & 77 & 118 & 258 \\
Median age (years) & $63(18-102)$ & $79(32-95)$ & $79(36-99)$ & $76(33-98)$ \\
Operative intervention, n (\%) & $295(35 \%)$ & $14(18 \%)$ & $34(29 \%)$ & $75(29 \%)$ \\
Average length of admission (days) & $17.2(1-247)$ & $10.9(1-95)$ & $10.7(1-49)$ & $12(1-174)$ \\
Average days in ICU/step down & $6.3(1-194)$ & $3.5(1-85)$ & $3.3(1-36)$ & $3.5(1-54)$ \\
Traumatic ICH, n (\%) & $402(48 \%)$ & $30(39 \%)$ & $56(47.5 \%)$ & $123(48 \%)$ \\
SDH, n (\%) & $367(44 \%)$ & $34(44 \%)$ & $63(53 \%)$ & $137(53 \%)$ \\
IPH, n (\%) & $368(44 \%)$ & $44(57 \%)$ & $52(44 \%)$ & $107(41 \%)$ \\
In-hospital mortality & $140(17 \%)$ & $29(38 \%)$ & $43(37 \%)$ & $58(22 \%)$ \\
Average mRS (0-6) & 3.2 & 3.4 & 3.6 & 3.4 \\
\hline
\end{tabular}


in about $80 \%$ at $15 \mathrm{~min}$, which increased to $100 \%$ at $30 \mathrm{~min}$. The reversal was sustained at $5-6 \mathrm{~h}$ in both groups. The most common treatment emergent AEs were sensations of warmth (hot flashes, flushing) which occurred in $40 \%$ but were mild, transient and dose-related.

Ciraparantag has several unique attributes in the current landscape of reversal agents. Being a small molecule rather than a biologic, it can be rapidly and easily administered and has the potential to be a universal reversal agent.

\section{COVID-19 and thrombosis}

COVID-19 associated coagulopathy is due to immunothrombosis [14], whereby a dysregulated immune system leads the activation of the coagulation cascade. The binding of SARS-CoV2 to pneumocytes in the alveoli results in immune cell infiltration and cytokine release which induces platelet aggregation and activation, complement activation and neutrophil extracellular traps, which contribute to the prothrombotic state seen with SARS-CoV2 infection. It has been established that the rate of VTE in higher in patients hospitalized COVID-19 compared to non-COVID and patients with COVID-19 who develop a VTE have a higher mortality compared to those who did not have any thromboembolic complications. $[15,16]$ The risk of VTE varies based upon the severity of illness and a recent metaanalysis showed that the pooled VTE prevalence in non-ICU patients was 7.9\% (95\% CI 5.1-11.20) and in ICU patients was $22.7 \%$ (95\% CI 18.1-27.6\%). [17] Additionally, there is equipoise among major guidelines regarding the optimal agent and dose thromboprophylaxis in patients admitted with COVID-19. Here we will discuss abstracts that focus on thromboprophylaxis in patients with COVID-19, both inpatient and post-discharge and quantify the burden of thrombosis in patients with COVID-19 in the outpatient setting and in those admitted with cancer and COVID-19.

\section{Intensified anticoagulation for hospitalized COVID-19 patients: admission Rothman index, aspirin, and intermediate dose anticoagulation effects on outcomes in COVID-19: a multi-site propensity matched analysis}

This was a retrospective cohort study to assess the impact of prophylactic aspirin or anticoagulation in patients hospitalized with COVID-19. [18] The overall cohort was divided into patients who received prophylactic or intermediate dose anticoagulation $(n=1624)$ and patients who received aspirin and were not on outpatient antiplatelet therapy. The investigators found that Rothman Index on admission was an independent predictor of mortality in the entire cohort along with older age, male sex, obesity and D-dimer. Among patients in the anticoagulation cohort, in-hospital mortality of patients who received intermediate dose anticoagulation $(n=191)$ was compared to those who received prophylactic dose anticoagulation $(n=191)$. Intermediate dose anticoagulation was associated with a decrease in mortality [OR 0.52 (0.31-0.87), $\mathrm{p}=0.013]$ compared to prophylactic dose. On comparing patients who received aspirin with those who did not $(\mathrm{n}=319 \mathrm{in}$ each), it was found that aspirin was associated with lower in-hospital mortality [OR $0.52(0.34-0.81)$, $\mathrm{p}=0.004]$.

\section{Empiric therapeutic anticoagulation for critically ill COVID-19 patients (Stop-COVID registry): thrombosis, bleeding, and the effect of anticoagulation on survival in critically III patients with COVID-19 in the United States}

A multicenter cohort of 3239 critically-ill patients with COVID-19 was analyzed to examine the effect of empiric therapeutic anticoagulation on 28-day survival. [19] The median age was 61 years (IQR 53-71), 64.5\% were male, $66 \%$ were intubated and $42 \%$ were on vasopressors on ICU day 1 . In the entire cohort of critically ill patients, 39.3\% died, $43.4 \%$ were discharged alive and $17.4 \%$ remained hospitalized at day 28 . The rate of radiographically confirmed VTE was $6.3 \%(n=204)$ and major bleeding was $2.8 \%(\mathrm{n}=90)$ during the first 14 days of ICU admission. The mortality in patients who developed a VTE was similar to the entire cohort but the mortality of patients who developed major bleeding was very high ( $71 \%$ at 28 days).

To determine the effect of therapeutic anticoagulation, patients who developed a confirmed or suspected VTE or had contraindications to therapeutic anticoagulation (major bleeding, thrombocytopenia) were excluded $(n=430)$. Of 2809 patients, $384(11.9 \%)$ received therapeutic anticoagulation within the first two days of ICU admission. At median follow-up of 27 days, the risk of death in those who received therapeutic anticoagulation was similar to those who did not (HR 1.12, 95\% CI 0.96-1.35) and there was no subgroup that benefitted from therapeutic anticoagulation.

\section{Anticoagulation after discharge: thromboembolic outcomes of hospitalized COVID-19 patients in the 90-day post-discharge period: early data from the northwell CORE-19 registry}

It is unknown whether the increased risk VTE in patients who have been hospitalized with COVID-19 persists after discharge and there is equipoise regarding the need for routine post-discharge thromboprophylaxis. Small retrospective studies have suggested that the risk of post-discharge VTE is similar to that after hospitalization for non-COVID acute medical illness. [20, 21] 
This study used a prospective registry (CORE-19) to assess the 90-day post discharge rate of VTE, ATE (arterial thromboembolism), bleeding and mortality in patients who had been hospitalized with COVID-19. [22] A total of 4906 patients were included, with mean age 61 years $( \pm 17.5)$, $54.7 \%$ were male, $10.8 \%$ had a personal history of VTE, $13 \%$ had a history of cancer. During hospitalization, 53.6\% received prophylactic enoxaparin, $20.1 \%$ received prophylactic unfractionated heparin (UFH) and $8.6 \%$ received prophylactic DOACs. At discharge, $1.4 \%$ were given prophylactic anticoagulation (enoxaparin in $0.9 \%$ ). The rate of VTE was $1.55 \%$ (DVT $0.9 \%$ and PE $0.85 \%$ ) and ATE was $1.71 \%$ in the 90-day follow up period. The risk of major bleeding was $1.73 \%$ and all-cause mortality was $4.83 \%$.

This large prospective study shows a higher risk of VTE post discharge than previously reported but seems to be similar to what has been observed in the control arms of randomized interventional studies $(0.3-2.5 \%)$. [23] It would be important to recognize the predictors of postdischarge thromboembolic events and find the appropriate population to conduct clinical trials for post-discharge thromboprophylaxis.

\section{COVID \& thrombosis in outpatients: incidence of vascular thromboembolic events in outpatients with mild COVID-19}

A retrospective analysis of 2673 outpatients diagnosed with COVID-19 was carried out to define the incidence of arterial and venous thromboembolic events within 30-days of diagnosis. [24] The mean age was 37.9 years $( \pm 17.3)$ and $54 \%$ were female. 20 patients $(0.7 \%)$ experienced a vascular thromboembolic event within 30 days of diagnosis, 3 patients had a VTE, 16 ATE and 1 patient experienced both. This study shows that non-hospitalized patients with COVID-19 tend to be younger and have a low risk of vascular thromboembolic events.

\section{COVID in cancer patients and the risk of thrombosis: incidence of and risk factors for venous thromboembolism among hospitalized patients with cancer and COVID-19: report from the COVID-19 and cancer consortium (CCC19) registry}

The thrombotic risk and predictors of VTE in hospitalized patients with cancer and COVID-19 is unknown. This was a retrospective cohort study from the $\mathrm{CCC} 19$ database, that analyzed the cumulative incidences of VTE, PE and arterial thromboembolic events (ATE) among hospitalized patients with cancer and laboratory confirmed SARS-CoV-2. [25] 1813 patients were included with a median age was 70 years (61-75) and median follow up of 51 days. 317 (20\%) were initially admitted to the ICU and $1249(80 \%)$ were initially admitted to the wards. The most common cancer types were prostrate (17\%), breast (14\%), gastrointestinal (14\%) and $39 \%$ had received anti-cancer therapy within 3 months of diagnosis of COVID-19. $9 \%$ patients had a prior history of VTE, pre-admission anticoagulant use was noted in 19\% and anti-platelet use in 33\% of the patients (Table 3).

The incidence of VTE was $8.8 \%$, PE was $5.1 \%$ and ATE was $3.9 \%$ in the entire cohort of hospitalized patients. Patients admitted to the ICU had a higher incidence of all thromboembolic events compared with those admitted to wards [OR 2.38 (95\% CI 1.63-3.42)]. The incidence of VTE was $13.9 \%$ versus $6.5 \%$, PE 9.8\% versus $3.4 \%$ and ATE $7.9 \%$ versus $2.9 \%$ in patients admitted to ICU and wards respectively. Recent anti-cancer therapy was associated with a higher risk of VTE and PE [OR 1.63 (95\% CI 1.02-2.44)] but was not associated with ATE. The subgroup of patients who had received recent anti-cancer therapy and were admitted to the ICU had the highest risk of developing VTE in the entire cohort (17.6\%). Pre-admission anticoagulant therapy and anti-platelet therapy seemed to be protective.

Even though this study had limitations, including the retrospective nature and lack of information about prophylactic anticoagulation, the results will aid in developing a risk prediction tool for VTE in patients with cancer who have been hospitalized with COVID-19.

In summary, these findings indicate that empiric therapeutic anticoagulation is not beneficial in severely ill patients

Table 3 Baseline characteristics of patients from the COVID-19 and cancer consortium (CCC19) registry

\begin{tabular}{ll} 
Total number of patients with cancer and COVID-19 & 1813 \\
Age, Median (IQR) & $70(61-79)$ \\
Initial admission & \\
ICU & $20 \%$ \\
Wards & $80 \%$ \\
Morbid obesity (BMI > 35-40) & $11 \%$ \\
History of VTE & $9 \%$ \\
Anticoagulant use pre-admission & $19 \%$ \\
Antiplatelet pre-admission & $33 \%$ \\
Recent anti-cancer therapy & $39 \%$ \\
Cancer remission status & \\
Remission & $46 \%$ \\
Active, stable/respond & $25 \%$ \\
Active, progressive & $16 \%$ \\
Unknown & $13 \%$ \\
Cancer subtypes & \\
Prostate & $17 \%$ \\
Breast & $14 \%$ \\
GI & $14 \%$ \\
Lymphoma & $12 \%$ \\
Thoracic & $9 \%$ \\
\hline
\end{tabular}


with COVID-19 and there is a high mortality associated with major bleeding. The role of intermediate dose prophylactic anticoagulation and prophylactic antiplatelet therapy still needs to be established. Managing the risk of bleeding and clotting in patients with COVID-19 admitted to the hospital is challenging and there are several ongoing trials which will help provide definite information. The low rate of thrombosis in patients with COVID-19 who are well enough to be outpatient and post-discharge suggests that the increased thrombotic risk is limited to patients with acute illness.

\section{Prevention of bleeding/thrombocytopenia/ ITP}

\section{Effects of tranexamic acid prophylaxis on bleeding outcomes in hematologic malignancy: the A-TREAT trial}

Tranexamic acid is an antifibrinolytic which has shown to reduce bleeding and death in post-partum hemorrhage, trauma and multiple surgical settings. Severe thrombocytopenia is common in patients undergoing treatment for hematological malignancies. However, despite optimal prophylactic platelet transfusions, the rate of WHO grade- 2 or higher bleeding is $43-70 \%$ (TOPSS trial and PLADO trial). Widespread endothelial damage in patients with hematological malignancies due to chemotherapy, GVHD and infections predisposes to clot formation. Usually when a clot is formed, platelets release a large amount of plasminogen activator inhibitor (PAI-1) which blocks the effect of tPA on plasminogen and stabilizes the blood clot. With thrombocytopenia, PAI-1 is not as abundant, leading to greater plasmin generation and faster clot degradation. Therefore, it was hypothesized that a fibrinolytic would efficacious in this setting.

The American Trial Using Tranexamic Acid (TXA) in Thrombocytopenia (A-TREAT) was a phase 3, doubleblinded, randomized, placebo-controlled trial to evaluate whether prophylactic tranexamic acid along with routine platelet transfusions would impact the bleeding and transfusion requirement in patients undergoing therapy for hematological malignancies. [26] 330 patients who were undergoing therapy for hematological malignancies and expected to have a platelet count of $<10 \mathrm{k} / \mu \mathrm{L}$ for $>5$ days were randomized. The mean age was 54.2 years $( \pm 13.1), 128$ patients underwent allotransplant, 72 underwent autologous transplant and 130 received chemo/immunotherapy. 165 patients received either intravenous $(1000 \mathrm{mg})$ or oral $(1300 \mathrm{mg})$ TXA and 165 received saline or placebo every $8 \mathrm{~h}$ beginning when the platelet count was $\leq 30 \mathrm{k} / \mu \mathrm{L}$ and was continued for 30-days or platelet recovery or a diagnosis of thrombosis. There was no significant difference in the proportion of grade 2 or higher bleeding ( $48.8 \%$ in placebo vs $45.4 \%$ in
TXA-group, OR 0.86 (95\% CI 0.52, 1.38; p value $=0.74$ )]. There was no significant difference in the mean number of transfusions [estimated difference 0.1 (95\% CI - 1.9, 2.0; $\mathrm{p}$ value $=0.94$ )] or average days alive without grade 2 or higher bleeding [average difference $0.1(95 \% \mathrm{CI}-1.4,1.5$; $\mathrm{p}$ value $=0.94)$ ]. However, there was an increase in the incidence thrombotic events TXA arm (11.0\% vs. $19.5 \%)$, most of which were central line occlusions. There was no increase in non-catheter thrombotic events.

This trial did not meet its primary end-point and conclusively proved that TXA is not useful in this setting. It is yet to be determined if TXA might have a role in a selected patient population like those with active bleeding or platelet refractoriness.

\section{A multicenter randomized trial of first line treatment pathways for newly diagnosed immune thrombocytopenia: standard steroid treatment versus combined steroid and mycophenolate. The FLIGHT trial}

Idiopathic thrombocytopenic purpura (ITP) is an acquired autoimmune disease which results in platelet destruction and impaired platelet production. ITP can lead to severe bleeding in $9.5 \%$ adults and significantly impair quality of life. [27] The recommended first line treatment is corticosteroids which lead to quick responses but durable remissions are seen in only $20 \%$. Mycophenolate (MMF) has been used as second-line treatment in some countries and is cheaper than most second-line treatment options. The FLIGHT trial evaluated whether addition of MMF to corticosteroids was better than the standard of care, corticosteroids alone, in patients with newly diagnosed ITP. [28]

This was a multicenter, open-label randomized control trial based in the UK. 120 patients were randomized to either corticosteroids and MMF $(n=59)$ or corticosteroids alone $(n=61)$. The corticosteroids included either dexamethasone $40 \mathrm{mg} \times 4$ days or prednisone $1 \mathrm{mg} / \mathrm{kg}$ followed by taper. MMF was started at $500 \mathrm{mg}$ BID with the option of escalating to $1000 \mathrm{mg}$ BID, was continued for 6 months and then tapered. At mean follow up of 18 months, the rate of treatment failures was significantly lower with MMF and corticosteroids compared to corticosteroids alone [22\% vs. 44\%; HR 0.41 (95\% CI 0.21-0.80), $\mathrm{p}=0.0064]$. Responses, defined as platelet count $>30 \mathrm{k} / \mu \mathrm{L}$ before second line treatment, were more frequent in the combination arm $(93.2 \%$ vs. $75.4 \%, \mathrm{p}=0.011)$ and fewer patients were refractory $(6.8 \%$ vs. $24.6 \%, p=0.011)$. There was no significant difference in the rate of AEs. Interestingly, patients in the combination arm scored worse on some aspects of quality of life including physical function and fatigue.

This study is the first randomized trial to demonstrate the safety and efficacy of using MMF in ITP. It also demonstrated 
that patient experience may not correspond to disease response and the importance of including patient reported outcome measures in clinical trials. Going forward, there would be a need identify biomarkers of corticosteroid refractoriness to identify patients who would benefit from upfront MMF.

\section{Oral rilzabrutinib, a Bruton tyrosine kinase inhibitor, showed clinically active and durable platelet responses and was well-tolerated in patients with heavily pretreated immune thrombocytopenia}

Rilzabrutinib is an oral, reversible, covalent inhibitor of Bruton tyrosine kinase (BTK) and is a novel approach for treating patients with ITP. BTK inhibition blocks antibody production by B-cells and phagocytosis in macrophages thereby targeting both the adaptive and innate drivers of immune-mediated disease. Additionally, it does not inhibit platelet aggregation unlike ibrutinib.

This was an open label Phase I/II study of rilzabrutinib in adults with relapsed/refractory ITP who had platelet count $<30 \mathrm{k} / \mu \mathrm{L}$. [29] It included patients with inadequate response to prior corticosteroids or thrombopoietin receptor agonists (TPO-RA) and allowed patients to continue receiving stable doses of these medications.

The minimal effective dose was determined to be $400 \mathrm{mg}$ BID from the dose-escalation phase. 38 patients were enrolled in the dose-expansion, the median age was 50 years (21-74) and median baseline platelet count was $17 \mathrm{k} / \mu \mathrm{L}$ (4-33). The median duration of ITP was 6 years $(0.4-52.5)$ and patients had received a median of 6 prior lines of therapies (1-53) including $24 \%$ who had prior splenectomy. 16 patients (42\%) achieved the primary endpoint (platelet count $\geq 50 \mathrm{k} / \mu \mathrm{L}$ and an increase of $\geq 20 \mathrm{k} / \mu \mathrm{L}$ from baseline without requiring rescue medications). The response was rapid and independent of prior therapies. 13 patients who had sustained response beyond 6 months entered the long-term extension (LTE) phase. These patients had durable response with platelet count $\geq 30 \mathrm{k} / \mu \mathrm{L}$ for $97 \%$ weeks on study. The median treatment duration was 19.6 weeks (1.4-24.6) for the entire cohort and 43.6 weeks (13.9-68.3) for LTE. TRAEs were observed in 47\%, of which diarrhea, nausea and fatigue were the most common. All of these were grade 1 or 2 and transient.

Rilzabrutinib represents a promising therapeutic strategy for patients with refractory and relapsed ITP. It was granted FastTrack designation by the FDA in October 2020 and Phase 3 studies are currently underway.

\section{Evaluating the role of gene therapy in hematologic disorders}

\section{Safety and efficacy of CTX001 in patients with transfusion-dependent $\beta$-thalassemia and sickle cell disease: early results from the climb THAL-111 and climb SCD-121 studies of autologous CRISPR-CAS9-modified CD34+ hematopoietic stem and progenitor cells}

The discovery the CRISPR-Cas9 nuclease system has moved the field of gene editing from basic science to a clinical reality. $\beta$-thalassemia and sickle cell disease are inherited hemoglobinopathies which arise from point mutations in the $\beta$ globin genes resulting in severe anemia, transfusion dependance, vaso-occlusive episodes (VOE), end-organ damage and high mortality. Most therapeutic options are geared towards improving symptoms but do not address the underlying cause of the disease. Gene editing represents a possible functional cure for patients with transfusion dependent $\beta$-thalassemia (TDT) and sickle cell disease (SCD).

Frangoul et al. used the CRISPR-Cas9 based gene-editing platform to alter the expression of genes that regulate fetal hemoglobin production in hematopoietic stem cells. [30] Elevated fetal Hb levels are known to decrease severity of TDT and SCD. The BCL11A gene encodes a key transcription factor which suppresses the production of fetal $\mathrm{Hb}$ and regulates the switch from fetal to adult $\mathrm{Hb}$ at birth. Therefore, silencing BCL11A reactivates gamma globin expression and induces fetal $\mathrm{Hb}$ production.

The Climb THAL-111 and Climb SCD-121 are Phase $1 / 2$, multicenter, single arm studies evaluating the safety and efficacy CTX001 in TDT and severe SCD. [31] In this study, peripheral CD34+ HSCs were mobilized, collected by apheresis and underwent ex vivo editing to reduce the erythroid specific expression of BCL11A to manufacture CTX001. Patients underwent myeloablative conditioning followed by infusion of CTX001. Results of the first 10 patients who had at least 3-month follow up was presented at ASH annual meeting 2020. Seven patients with TDT had a median age of 23 years (19-26) and required a median of 33 units of RBCs/year (23-61). Post infusion, all patients demonstrated clinically meaningful elevation of total and fetal $\mathrm{Hb}$ and achieved transfusion independence. The median $\mathrm{Hb} 11.5 \mathrm{~g} / \mathrm{dL}$ and the median fetal $\mathrm{Hb}$ was $8.4 \mathrm{~g} / \mathrm{dL}$ in 7 patients who had a 3-month follow up and this response improved with time as demonstrated by transfusion independence in 1 patient at 21 months. There was pan-cellular expression of fetal $\mathrm{Hb}$ at 6 months with 99\% circulating RBCs expressing HbF. Three patients with severe SCD with median age of 22 years had a median 7 
VOEs in the past year (4-7.5). There was early and sustained improvement in total and fetal $\mathrm{Hb}$. At last follow up (3.8-16.6 months), there were no episodes of VOEs, the fetal $\mathrm{Hb}$ ranged from 31 to $48 \%$ and total $\mathrm{Hb} 11.5-13.2$.

The majority of the adverse events occurred within 60 days and were consistent with myeloablative conditioning and autologous transplant. This study is very encouraging and provides a proof of principle of the clinical potential of gene-editing treatments.

\section{First data from the phase 3 HOPE-B gene therapy trial: efficacy and safety of etranacogene dezaparvovec (AAV5-Padua hFIX variant; AMT-061) in adults with severe or moderate-severe hemophilia B treated irrespective of pre-existing anti-capsid neutralizing antibodies}

Severe hemophilia is characterized by high risk of spontaneous bleeding, especially hemarthrosis requiring prophylactic administration of Factor IX concentrates. Etranacogene dezaparvovec (AAV5-Padua hFIX; AMT-061) is an investigational gene therapy for Hemophilia B which consists of combining an adenoviral vector (AAV5) with the Padua variant human factor IX gene. The naturally occurring Padua FIX variant is 8-times more active that wild type FIX. The goal of gene therapy for severe hemophilia B is sustained increase FIX activity to mild/normal levels after a one-time procedure which would decrease the risk of bleeding, improve the quality of life and eliminate the need for continuous prophylaxis.

The HOPE-B (Health Outcomes with Padua gene; Evaluation in Hemophilia B) was an open-label, single-arm multicenter Phase 3 trial to establish the efficacy and safety of etranacogene dezaparvovec in adult males with Hemophilia B. [32] 54 patients with moderate-severe or severe hemophilia (Factor IX $\leq 2 \%$ ) were treated with a single dose, regardless of the presence of neutralizing antibodies (nAbs) to AVV5 and had completed 26 weeks follow up. The mean age was 41.5 years $( \pm 15.8), 38$ patients $(70.4 \%)$ had a total of 123 bleeds during the 6-month lead in despite prophylaxis and 23 (42.6\%) had pre-existing nAbs to AAV5. The FIX activity increased to a mean to $37.2 \%$ ( \pm 19.6 ; range 1-97.1) post-treatment, which represented a change of $36 \%$ ( \pm 19.7, range 0-96.1) from baseline. In the 26-week follow up period, 39 patients (72.2\%) had no bleeds and 15 patients reported a total of 21 bleeds. $96.3 \%$ were able to successfully discontinue routine prophylaxis.

37 patients $(68.5 \%)$ had treatment related adverse events (TRAEs), the majority of which were mild. 9 patients received steroid treatment for transaminase elevation, 7 experienced infusion related reactions and infusion had to be discontinued in 1 patient.
The HOPE-B is the first Phase 3 trial in Hemophilia $\mathrm{B}$ and the largest gene therapy cohort to date. It was also unique in that patients with pre-existing anti-neutralizing antibodies were included and responded to treatment.

In summary, our review of abstracts from ASH, 2020 focuses on important studies around non-malignant hematology topics that would implicate change of practice in the years to come. There are certainly several more studies, which we encourage the readers to review from ASH.

Author contributions Both SG and PG collected data, conceptualized the manuscript, wrote and edited the manuscript.

Funding The authors did not receive support from any organization for the submitted work.

Data availability Data gathered from meeting materials.

\section{Declarations}

Conflict of interest The authors have no relevant financial or non-financial interests to disclose.

Consent to publication Yes.

Research involving human rights This review does not report data on human subject research.

\section{References}

1. Davenport A (2011) What are the anticoagulation options for intermittent hemodialysis? Nat Rev Nephrol 7(9):499-508

2. Lorentz CU, Verbout NG, Shatzel JJ et al (2020) Safety and efficacy of the contact activation inhibitor $\mathrm{AB} 023$ in patients with end-stage renal disease on chronic hemodialysis: A phase 2, double-blind, randomized placebo-controlled trial. Blood 136(Supplement 1):23-24

3. Khorana AA, Kuderer NM, Culakova E et al (2008) Development and validation of a predictive model for chemotherapy-associated thrombosis. Blood 111(10):4902-4907

4. Iorio A, Kearon C, Filippucci E et al (2010) Risk of recurrence after a first episode of symptomatic venous thromboembolism provoked by a transient risk factor: a systematic review. Arch Intern Med 170(19):1710-1716

5. Kapoor S, Opneja A, Gollamudi J et al (2020) Prior history of venous thromboembolism is a significant risk factor for recurrence of thrombosis after cancer diagnosis. Blood 136(Supplement 1):32-33

6. Eichinger S, Hron G, Bialonczyk C et al (2008) Overweight, obesity, and the risk of recurrent venous thromboembolism. Arch Intern Med 168(15):1678-1683

7. McCaughan GJ, Crowther HJ, Chen V et al (2020) Australian and New Zealand registry of anticoagulation in the obese: prescribing patterns, drug levels and patient outcomes. Blood 136(Supplement 1):21-21

8. Hart RG, Diener HC, Yang S et al (2012) Intracranial hemorrhage in atrial fibrillation patients during anticoagulation with warfarin or dabigatran: the RE-LY trial. Stroke 43(6):1511-1517 
9. Hankey GJ, Stevens SR, Piccini JP et al (2014) Intracranial hemorrhage among patients with atrial fibrillation anticoagulated with warfarin or rivaroxaban: the rivaroxaban once daily, oral, direct factor Xa inhibition compared with vitamin $\mathrm{K}$ antagonism for prevention of stroke and embolism trial in atrial fibrillation. Stroke 45(5):1304-1312

10. Srinathan S, Cipkar C, Chiang P et al (2020) Mortality rate and functional outcomes among patients presenting with anticoagulation- associated intracranial hemorrhage. Blood 136(Supplement 1):24-25

11. Inohara T, Xian Y, Liang L et al (2018) Association of intracerebral hemorrhage among patients taking non-vitamin K antagonist vs vitamin $\mathrm{K}$ antagonist oral anticoagulants with in-hospital mortality. JAMA 319(5):463-473

12. Tsivgoulis G, Katsanos AH, Seiffge DJ et al (2020) Fatal intracranial haemorrhage occurring after oral anticoagulant treatment initiation for secondary stroke prevention in patients with atrial fibrillation. Eur J Neurol 27(8):1612-1617

13. Ansell J, Laulicht B, Bakhru S et al (2020) Reversal of anticoagulation by ciraparantag: time to onset and duration of effect. Blood 136(Supplement 1):24-24

14. Loo J, Spittle DA, Newnham M (2021) COVID-19, immunothrombosis and venous thromboembolism: biological mechanisms. Thorax 76(4):412-420

15. Poissy J, Goutay J, Caplan M et al (2020) Pulmonary embolism in patients with COVID-19. Circulation 142(2):184-186

16. Malas MB, Naazie IN, Elsayed N et al (2020) Thromboembolism risk of COVID-19 is high and associated with a higher risk of mortality: a systematic review and meta-analysis. EClinicalMedicine. https://doi.org/10.1016/j.eclinm.2020.100639

17. Nopp S, Moik F, Jilma B et al (2020) Risk of venous thromboembolism in patients with COVID-19: a systematic review and meta-analysis. Res Pract Thromb Haemost 4(7):1178-1191

18. Goshua G, Liu Y, Meizlish ML et al (2020) Admission Rothman index, aspirin, and intermediate dose anticoagulation effects on outcomes in COVID-19: a multi-site propensity matched analysis. Blood 136(Supplement 1):23-24

19. Al-Samkari H, Gupta S, Karp Leaf R et al (2020). Thrombosis, bleeding, and the effect of anticoagulation on survival in critically ill patients with COVID-19 in the United States [abstract]. American Society of Hematology Annual Meeting

20. Patell R, Bogue T, Koshy A et al (2020) Postdischarge thrombosis and hemorrhage in patients with COVID-19. Blood 136(11):1342-1346

21. Roberts LN, Whyte MB, Georgiou L et al (2020) Postdischarge venous thromboembolism following hospital admission with COVID-19. Blood 136(11):1347-1350

22. Giannis D, Allen SL, Davidson A et al (2020) Thromboembolic outcomes of hospitalized COVID-19 patients in the 90-day postdischarge period: early data from the northwell CORE-19 registry. Blood 136(Supplement 1):33-34
23. Bhalla V, Lamping OF, Abdel-Latif A et al (2020) Contemporary meta-analysis of extended direct-acting oral anticoagulant thromboprophylaxis to prevent venous thromboembolis. Am J Med 133(9):1074-1081.e1078

24. Eswaran H, Jarmul J, Shaheen A et al (2020) Incidence of vascular thromboembolic events in outpatients with mild COVID-19. Blood 136(Supplement 1):31-32

25. Li A, Kuderer NM, Warner JL et al (2020) Incidence of and risk factors for venous thromboembolism among hospitalized patients with cancer and COVID-19: report from the COVID-19 and cancer consortium (CCC19) registry. Blood 136(Supplement 1):56-58

26. Gernsheimer TB, Brown SP, Triulzi DJ et al (2020) Effects of tranexamic acid prophylaxis on bleeding outcomes in hematologic malignancy: the a-TREAT trial. Blood 136(Supplement 1):1-2

27. Neunert C, Noroozi N, Norman G et al (2015) Severe bleeding events in adults and children with primary immune thrombocytopenia: a systematic review. J Thromb Haemost 13(3):457-464

28. Bradbury CA, Greenwood R, Pell J et al (2020) A multicentre randomised trial of first line treatment pathways for newly diagnosed immune thrombocytopenia: standard steroid treatment versus combined steroid and mycophenolate. The flight trial. Blood 136(Supplement 2):LBA-2

29. Kuter DJ, Efraim M, Mayer J et al (2020) Oral rilzabrutinib, a bruton tyrosine kinase inhibitor, showed clinically active and durable platelet responses and was well-tolerated in patients with heavily pretreated immune thrombocytopenia. Blood 136(Supplement 1):13-14

30. Frangoul H, Altshuler D, Cappellini MD et al (2020) CRISPRCas9 gene editing for sickle cell disease and $\beta$-thalassemia. $\mathrm{N}$ Engl J Med 384(3):252-260

31. Frangoul H, Bobruff Y, Cappellini MD et al (2020) Safety and efficacy of CTX001 in patients with transfusion-dependent $\beta$-thalassemia and sickle cell disease: early results from the climb THAL-111 and climb SCD-121 studies of autologous CRISPRCAS9-modified CD34+ hematopoietic stem and progenitor cells. Blood 136(Supplement 1):3-4

32. Pipe SW, Recht M, Key NS et al (2020) First data from the phase 3 HOPE-B gene therapy trial: efficacy and safety of etranacogene dezaparvovec (AAV5-Padua hFIX variant; AMT-061) in adults with severe or moderate-severe hemophilia B treated irrespective of pre-existing anti-capsid neutralizing antibodies. Blood 136(Supplement 2):LBA-6

Publisher's Note Springer Nature remains neutral with regard to jurisdictional claims in published maps and institutional affiliations. 